\title{
Micron-sized forsterite grains in the pre-planetary nebula of IRAS 17150-3224
}

\section{Searching for clues to the mysterious evolution of massive AGB stars}

\author{
B. L. de Vries ${ }^{1,2}$, K. M. Maaskant ${ }^{3}$, M. Min $^{4}$, R. Lombaert ${ }^{5}$, L. B. F. M. Waters ${ }^{4,6}$, and J. A. D. L. Blommaert ${ }^{5,7}$ \\ 1 AlbaNova University Centre, Stockholm University, Department of Astronomy, 10691 Stockholm, Sweden \\ e-mail: bernard.devries@astro.su.se, bldevries.science@gmail.com \\ 2 Stockholm University Astrobiology Centre, 10691 Stockholm, Sweden \\ 3 Leiden Observatory, Leiden University, PO Box 9513, 2300 RA Leiden, The Netherlands \\ 4 Anton Pannekoek Astronomical Institute, University of Amsterdam, PO Box 94249, 1090 GE Amsterdam, The Netherlands \\ ${ }^{5}$ Instituut voor Sterrenkunde, KU Leuven, Celestijnenlaan 200D, 3001 Leuven, Belgium \\ ${ }^{6}$ SRON Netherlands Institute for Space Research, Sorbonnelaan 2, 3584 CA Utrecht, The Netherlands \\ 7 Department of Physics and Astrophysics, Vrije Universiteit Brussel, Pleinlaan 2, 1050 Brussels, Belgium
}

Received 21 August 2014 / Accepted 17 February 2015

\section{ABSTRACT}

\begin{abstract}
Aims. We study the grain properties and location of the forsterite crystals in the circumstellar environment of the pre-planetary nebula (PPN) IRAS 17150-3224 in order to learn more about the as yet poorly understood evolutionary phase prior to the PPN.

Methods. We use the best-fit model for IRAS 17150-3224 of Meixner et al. (2002, ApJ, 571, 936) and add forsterite to this model. We investigate different spatial distributions and grain sizes of the forsterite crystals in the circumstellar environment. We compare the spectral bands of forsterite in the mid-infrared and at $69 \mu \mathrm{m}$ in radiative transport models to those in ISO-SWS and Herschel/ PACS observations.

Results. We can reproduce the non-detection of the mid-infrared bands and the detection of the $69 \mu \mathrm{m}$ feature with models where the forsterite is distributed in the whole outflow, in the superwind region, or in the AGB-wind region emitted previous to the superwind, but we cannot discriminate between these three models. To reproduce the observed spectral bands with these three models, the forsterite crystals need to be dominated by a grain size population of $2 \mu \mathrm{m}$ up to $6 \mu \mathrm{m}$. We also tested models where the forsterite is located in a torus region or where it is concentrated in the equatorial plane, in a disk-like fashion. These models show either absorption features that are too strong or a $69 \mu \mathrm{m}$ band that is too weak, respectively, so we exclude these cases. We observe a blue shoulder on the $69 \mu \mathrm{m}$ band that cannot be explained by forsterite and we suggest a possible population of micron-sized ortho-enstatite grains. We hypothesise that the large forsterite crystals were formed after the superwind phase of IRAS 17150-3224, where the star developed an as yet unknown hyperwind with an extremely high mass-loss rate $\left(\gtrsim 10^{-3} M_{\odot} / \mathrm{yr}\right)$. The high densities of such a hyperwind could be responsible for the efficient grain growth of both amorphous and crystalline dust in the outflow. Several mechanisms are discussed that might explain the lower-limit of $\sim 2 \mu \mathrm{m}$ found for the forsterite grains, but none are satisfactory. Among the mechanisms explored is a possible selection effect due to radiation pressure based on photon scattering on micron-sized grains.
\end{abstract}

Key words. radiative transfer - stars: winds, outflows - planetary nebulae: general - stars: AGB and post-AGB - stars: evolution stars: mass-loss

\section{Introduction}

IRAS 17150-3224 (also known as AFGL 6815 or $\mathrm{OH} 353.84+2.98$ ) is a pre-planetary nebula (PPN), characterised by a stellar remnant surrounded by a previously expelled dust and gas envelope. The infrared emission from the dust envelope of IRAS 17150-3224 has been studied by Guertler et al. (1996), who modelled the spectral energy distribution (SED) of that source up to wavelengths of $1.3 \mathrm{~mm}$. Using a spherical geometry for the dust envelope they found that they needed either large grains or a second cold shell in order to properly fit the far-infrared emission of IRAS 17150-3224. Based on Hubble resolved images, Ueta et al. (2000) and Meixner et al. (1999) later showed that the envelope of IRAS 17150-3224 is axisymmetric. Meixner et al. (2002) modelled both the SED and the resolved images using an axisymmetric dust distribution and amorphous grains with a minimum grain size of $0.001 \mu \mathrm{m}$ and a maximum size of up to sizes of several hundred micrometres.
The axisymmetric envelope of IRAS $17150-3224$ is the material lost by IRAS 17150-3224 during its asymptotic giant branch (AGB) phase. The axisymmetric morphologies are also recognised in planetary nebulae themselves and these morphologies are thought to predate the PPN phase.

IRAS $17150-3224$ is likely an evolutionary product of a massive $\left(\gtrsim 5 M_{\odot}\right)$ main-sequence star (Meixner et al. 2002). This links IRAS 17150-3224 to stars that in their AGB phase stay oxygen-rich. Such massive AGB stars have mass-loss rates of the order of $\sim 10^{-4} M_{\odot} / \mathrm{yr}$ when in their $\mathrm{OH} / \mathrm{IR}$ phase (Vassiliadis $\&$ Wood 1993). This period of high mass-loss is often called the superwind (Iben \& Renzini 1981; Knapp \& Morris 1985; Bedijn 1987; Wood et al. 1992).

Even though the superwind of massive AGB stars was introduced so that the central star could lose enough mass to reach white dwarf masses, it is now becoming increasingly clear that the superwind is not capable of shedding enough mass. 
The superwind timescale turns out to be too short ( $<2000 \mathrm{yrs})$ and the number of superwind phases seems to be limited to only one. These short timescales have been shown with several methods, either based on gas and SED modelling (Heske et al. 1990; Justtanont et al. 2006, 2013, 1996; Decin et al. 2007; Chesneau et al. 2005; Groenewegen 2012) or based on the analysis of forsterite spectral features (de Vries et al. 2014).

These short timescales pose an evolutionary problem. The mass loss rates of massive AGB stars can go up to $15 \times 10^{-5} M_{\odot} / \mathrm{yr}$ and even slightly higher (Justtanont et al. 1996; Justtanont \& Tielens 1992; Schutte \& Tielens 1989; Groenewegen 1994). Such mass-loss rates, combined with the found timescales, gives a total mass lost in one superwind of $\sim 0.2-0.6 M_{\odot}$ (depending on the gas over dust ratio of 100-300, Lombaert et al. 2013). Massive AGB stars need to lose several solar masses before they can leave the AGB. Therefore one would expect to see several superwind phases as extended shells around massive AGB stars and their remnants, in the same way as extended shells are seen around carbon-rich AGB stars (Cox et al. 2012; Maercker et al. 2012). It is intriguing that even though several oxygen-rich AGB stars have been observed with Herschel, none of them show any extended structure (Cox et al. 2012). It is likely that this is an indication that massive AGB stars have no extended shells and thus no previously ejected superwinds. It is then hard to understand how these massive AGB stars lose enough mass to evolve away from the AGB into a white dwarf.

de Vries et al. (2014) hypothesise an intermittent phase between the PPN phase and the superwind, where the star has a mass-loss rate one or two orders higher than during the superwind. This phase of extreme mass-loss could be referred to as the hyperwind. With hyperwind mass-loss rates of the order of $10^{-3}-10^{-2} M_{\odot} / \mathrm{yr}$, several hundreds to thousands of years would be enough for the central star to evolve into a white dwarf.

The central star of a massive AGB during its superwind is already completely obscured and its SED is very red, peaking at $\sim 30 \mu \mathrm{m}$. The SED of a star in its hyperwind phase would be even redder and would peak at even longer wavelengths, while its central star would still be totally obscured. So far, stars in their hyperwind phase have not been observed or identified, because the phase is so short, the superwind is still too optically thick to see the hyperwind through it, or such sources might have been wrongly classified (possibly as protostellar). However hints of the hyperwind can already be seen in the models of IRAS 17150-3224 and other post-AGB sources (e.g. IRAS 16432-3814 in Dijkstra et al. 2003 or IRAS 18276-1431 in Murakawa et al. 2013). Meixner et al. (2002) show that, at its highest, the mass-loss rate of IRAS 17150-3224 must have been of the order of $8.5 \times 10^{-3} M_{\odot} / \mathrm{yr}$. This makes IRAS 17150-3224 a crucial object to study in the light of this evolutionary conundrum.

Another property that links IRAS 17150-3224 to massive AGB stars is the presence of forsterite $\left(\mathrm{Mg}_{2} \mathrm{SiO}_{4}\right)$ in its outflow. Massive AGB stars in their superwind phase show prominent forsterite features at among others 11.3, 33.6 and $69 \mu \mathrm{m}$ (Waters et al. 1996; Molster et al. 2002; de Vries et al. 2010, 2014; Blommaert et al. 2014). The abundance of forsterite in these superwinds can be as high as $14 \%$ by mass (de Vries et al. $2010,2014)$. Since the spectrum of IRAS 17150-3224 does not have any convincing mid-infrared spectral features of crystalline dust species (Meixner et al. 2002, see Fig. 1), it was thought that the outflow of IRAS 17150-3224 contains no crystalline dust component, but recently Blommaert et al. (2014) reported the detection of the far-infrared spectral band at $69 \mu \mathrm{m}$ of forsterite.

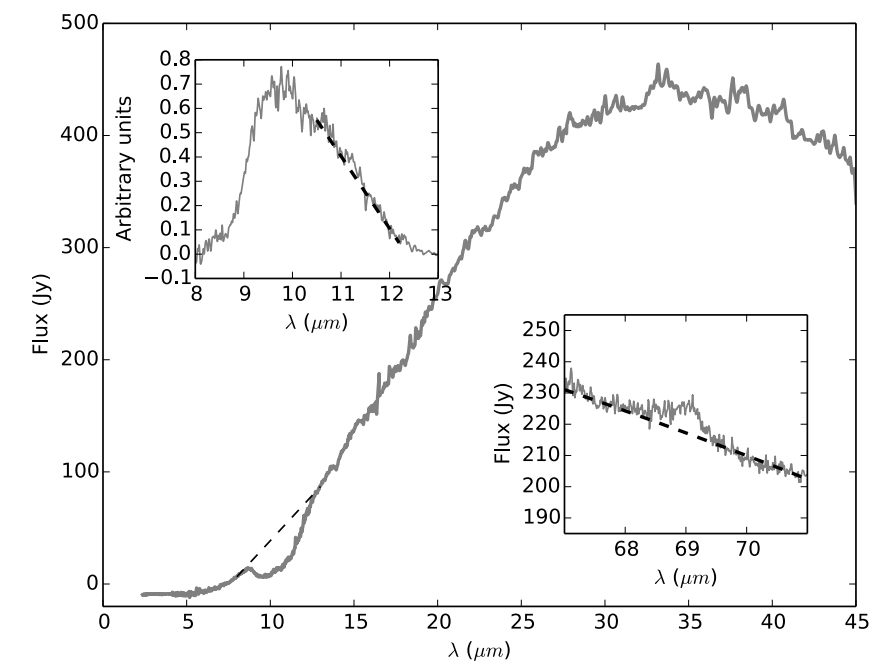

Fig. 1. Main window: ISO-SWS spectrum of IRAS 17150-3224 in grey (Sloan et al. 2003). The continuum constructed over the $9.7 \mu \mathrm{m}$ band of amorphous olivine (as explained in Sect. 4.3) is shown as a dashed black line. Top-left inset: optical depth of the $9.7 \mu \mathrm{m}$ absorption feature (see Sect. 4.3) in grey and in dashed black the continuum under the $11.3 \mu \mathrm{m}$ band of forsterite. Bottom right inset: $69 \mu \mathrm{m}$ band as observed with Herschel/PACS in grey (Blommaert et al. 2014) and the constructed continuum under the $69 \mu \mathrm{m}$ band in dashed black.

The study of the sharp spectral bands of forsterite is of great value since these spectral bands are dependent on many properties of the grains (see Sect. 3) and thus on the outflow in general. As we will show in this work, a special combination of parameters is needed for IRAS 17150-3224 in order to explain both the absence of forsterite features in the mid-infrared and a strong detection at $69 \mu \mathrm{m}$. To this end we use radiative transport models.

In this paper we start by introducing the observations in Sect. 2. The behaviour of the different mid- and far-infrared bands of forsterite are described in Sect. 3. Section 4 contains the modelling strategy of this work, the radiative-transport code we use and how we study the spectral features in the model and observed spectra. We continue in Sect. 5 with the results and end the paper with a discussion in Sect. 6 and our conclusions in Sect. 7.

\section{Observations}

Most recently Meixner et al. (2002) have modelled the SED and images of IRAS 17150-3224. In their fitting they have included the $B$-band Hubble Space Telescope image from Ueta et al. (2000), the $9.8 \mu \mathrm{m}$ images from Meixner et al. (1999) and the optical and infrared photometry of a compilation of data made by Ueta et al. (2000) (including data from van der Veen et al. 1989; Hu et al. 1993; Kwok et al. 1996; Reddy \& Parathasarathy 1996; Meixner et al. 1999; Ueta et al. 2000; and IRAS). Meixner et al. (2002) were able to reproduce the SED and images of IRAS 17150-3224 with an axisymmetric density distribution and by including a population of amorphous olivine grains with a size distribution from $0.001 \mu \mathrm{m}$ up to $\sim 200 \mu \mathrm{m}$ and then falling off exponentially.

We will build on the results of Meixner et al. (2002) by studying the now known presence of forsterite in the outflow of IRAS 17150-3224. For this we use the ISO-SWS spectrum of IRAS 17150-3224 (Sloan et al. 2003, see Fig. 1). The ISO-SWS spectrum shows a very smooth dust continuum and a $9.7 \mu \mathrm{m}$ absorption band, which are both due to amorphous silicates. As 
mentioned by Meixner et al. (2002) the ISO-SWS spectrum contains no clear indications of any crystalline silicate bands (for example at $11.3 \mu \mathrm{m}$ or $33.6 \mu \mathrm{m}$ ). The presence of three weak emission features at $33.6 \mu \mathrm{m}, \sim 38 \mu \mathrm{m}$, and $\sim 41 \mu \mathrm{m}$ could be argued, but this is uncertain at the end of the wavelength range of ISO-SWS. The fact that an absorption band of amorphous silicates is seen at $9.7 \mu \mathrm{m}$ while no absorption band of forsterite is seen at $11.3 \mu \mathrm{m}$ is strikingly different from that seen in observations of massive AGB stars, the likely predecessor phase of IRAS 17150-3224.

In contrast to the absence of mid-infrared features, the farinfrared spectrum of IRAS 17150-3224 shows a clear $69 \mu \mathrm{m}$ band of forsterite (Blommaert et al. 2014, see Fig. 1). In the farinfrared we use the Herschel/PACS observations of the $69 \mu \mathrm{m}$ (Groenewegen et al. 2011; Blommaert et al. 2014) band. The detection of the $69 \mu \mathrm{m}$ band in IRAS 17150-3224 was reported by Blommaert et al. (2014) to be broader than could be explained from its central wavelength position. Blommaert et al. (2014) showed that a temperature gradient for the forsterite component was not enough to explain the width of the $69 \mu \mathrm{m}$ band of IRAS 17150-3224. In Sect. 3 we will explain in more detail how the band properties of the $69 \mu \mathrm{m}$ band depend on grain temperature and grain size.

In this work we will study the combination of the nondetection of features in the mid-infrared together with the detection of the $69 \mu \mathrm{m}$ band. We will also show that the broadness of the $69 \mu \mathrm{m}$ band as reported by Blommaert et al. (2014) is due to a shoulder on the blue side of the $69 \mu \mathrm{m}$ band, which cannot be due to forsterite.

\section{Forsterite features}

As many different investigations have shown, the spectral features of forsterite contain a wealth of information (Koike et al. 2003; Suto et al. 2006; Bowey et al. 2002; Sturm et al. 2010, 2013; Mulders et al. 2011; de Vries et al. 2012, 2014; Maaskant et al. 2015; Blommaert et al. 2014). Much of this information is contained in the $69 \mu \mathrm{m}$ band, whose shape is strongly dependent on the grain temperature, the iron content of the crystal and, for micron-sized grains, also on the crystal size. The band broadens and its central wavelength position shifts to the red as either the temperature increases, the iron content increases or the grain size becomes larger than $\sim 7 \mu \mathrm{m}$ in size (Koike et al. 2003; Suto et al. 2006; Sturm et al. 2013; Maaskant et al. 2015). Figure 2 shows the behaviour of the $69 \mu \mathrm{m}$ band as a function of grain size. It can be seen that the effect of grain size on the $69 \mu \mathrm{m}$ band is small below grain sizes of $7 \mu \mathrm{m}$, while above this limit the $69 \mu \mathrm{m}$ band broadens significantly as a function of grain size.

The bands of forsterite in the region of the $9.7 \mu \mathrm{m}$ band of amorphous silicates, like the $11.3 \mu \mathrm{m}$ band, originate from degrees of freedom within the $\mathrm{SiO}_{4}$ tetrahedral and they are therefore not sensitive to the composition of the crystalline olivine (Koike et al. 2003). By approximation, these bands are also not sensitive to the grain temperature (Zeidler 2012). The forsterite bands in the mid-infrared (like for example the $33.6 \mu \mathrm{m}$ band, see Fig. 2) are, compared to the $69 \mu \mathrm{m}$ band, mildly dependent on the grain temperature and composition (Koike et al. 2003; Zeidler 2012).

All spectral bands of forsterite are dependent on grain size, but at which grain size their dependence becomes significant depends on the wavelength position of the band. The grain size dependence of the bands comes from the fact that at a certain size, the interior of the grain is not sampled by the radiation field
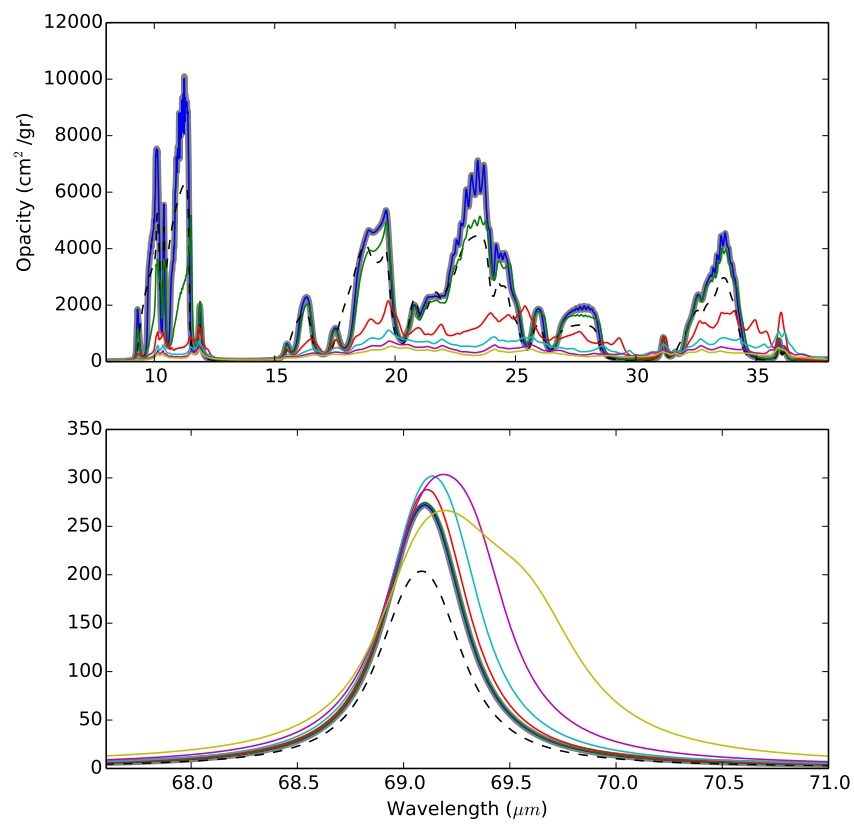

Fig. 2. Effect of grain size on the mid-infrared and $69 \mu \mathrm{m}$ bands of $150 \mathrm{~K}$ forsterite grains. The grey, blue, green, red, teal, purple, and yellow curves are for forsterite with grain sizes of $0.001,0.1,1,3,5$, 7 and $9 \mu \mathrm{m}$, respectively. The opacities are calculated for grains with a DHS shape distribution (Min et al. 2005), using the optical constants of Suto et al. (2006). The dashed black line are the opacities when calculated with CDE (Continuous Distribution of Ellipsoids; Bohren \& Huffman 1983).

anymore, causing the band to broaden and eventually to disappear. As is shown in Fig. 2, already at grain sizes of $1 \mu \mathrm{m}$ the band at $11.3 \mu \mathrm{m}$ becomes weaker as a function of grain size. At a grain size of $1 \mu \mathrm{m}$ this is not yet the case, causing the band at that wavelength to still be quite insensitive to grain size. Eventually the $69 \mu \mathrm{m}$ band will be influenced by the grain size when the grain size becomes larger than $\sim 7 \mu \mathrm{m}$.

Because of the grain size dependence, the peak ratios of the spectral bands are a good probe of the grain size. This was recently shown and used by Maaskant et al. (2015). They found $\sim 10 \mu \mathrm{m}$ forsterite crystals in the proto-planetary disk of HD 141569 by studying the relative strengths of the $69 \mu \mathrm{m}$ band compared to mid-infrared bands. However, before doing such a grain size analysis, a good model for the temperature and density structure of the circumstellar environment needs to be known. For IRAS 17150-3224, a satisfactory model was derived by Meixner et al. (2002), which provides us with the needed parameters to calculate the optical depth, density and temperature of the envelope in order to study the forsterite spectral signature (see Sect. 4.2).

\section{Method}

\subsection{Strategy}

Here we discuss three ways by which the spectrum of forsterite dust would only show a $69 \mu \mathrm{m}$ band and no features in the midinfrared. The first is that the material is very cold and the temperature of the material has a black-body spectrum that peaks at the far-infrared wavelengths or slightly beyond. A second option is that the medium has an optical depth that causes all the midinfrared features to be exactly in between absorption and emission. The third option is the presence of micron-sized forsterite 
Table 1. Best-fit model parameters of Meixner et al. (2002).

\begin{tabular}{lc}
\hline \hline \multicolumn{2}{l}{ Meixner et al. (2002) fit parameters } \\
\hline$L_{\text {star }}\left(L_{\odot}\right)$ & 27200 \\
$T_{\text {star }}(\mathrm{K})$ & 5200 \\
$R_{\text {star }}\left(R_{\odot}\right)$ & 201 \\
Distance $(\mathrm{kpc})$ & 3600 \\
$R_{\text {in }}(\mathrm{AU})$ & 648.4 \\
$R_{\text {Sw }}(\mathrm{AU})$ & 1944.4 \\
$R_{\text {out }}(\mathrm{AU})$ & $64 \times 10^{3}$ \\
$v_{\text {exp }}\left(\mathrm{km} \mathrm{s}^{-1}\right)$ & 15 \\
Inclination $\left.^{\circ}{ }^{\circ}\right)$ & 82.0 \\
Total dust mass $\left(M_{\odot}\right)$ & $4.6 \times 10^{-2}$ \\
$A_{\text {meix }}$ & 159 \\
$B_{\text {meix }}$ & 2.0 \\
$C_{\text {meix }}$ & 1.5 \\
$D_{\text {meix }}$ & 1.0 \\
$E_{\text {meix }}$ & 4.0 \\
$F_{\text {meix }}$ & 1.5 \\
Am. grain size $(\mu \mathrm{m})$ & $0.001-200$ \\
Am. grain size slope & -3.5 \\
\hline
\end{tabular}

Table 2. Forsterite abundances used for the models in Fig. 3.

\begin{tabular}{|c|c|c|c|c|c|c|}
\hline \multicolumn{7}{|c|}{ Forsterite abundance (\%, see Sect. 4.1$)$} \\
\hline Grain size $(\mu \mathrm{m})$ : & $<0.1$ & 1 & 2 & 4 & 6 & 8 \\
\hline Whole & 1.3 & 1.3 & 1.3 & 1.6 & 1.8 & 2.1 \\
\hline Superwind & 3.8 & 3.8 & 3.8 & 3.8 & 3.8 & 3.8 \\
\hline$A G B$-wind & 1.6 & 1.6 & 1.6 & 2.6 & 3.4 & 4.8 \\
\hline Torus & 10.6 & 10.6 & 10.6 & 11.5 & 12.3 & 11.9 \\
\hline Disk & 90.0 & - & - & - & - & - \\
\hline
\end{tabular}

crystals ( $\gtrsim 1 \mu \mathrm{m})$, which do not show spectral bands at short wavelengths because the grain size is significantly larger than the wavelength, but still have the $69 \mu \mathrm{m}$ band in the far-infrared.

In order to find out which of the three options is the cause for the peculiar forsterite spectrum of IRAS 17150-3224, we study forsterite features over a wide range of wavelengths, from $11.3 \mu \mathrm{m}$ up to the $69 \mu \mathrm{m}$ band. We choose features situated at wavelengths far apart, which helps us to probe the largest possible ranges in optical depth and temperature. The $11.3 \mu \mathrm{m}$ band is very sensitive to the grain size in the range between $0.1 \mu \mathrm{m}$ and $5.0 \mu \mathrm{m}$, but not to the grain temperature and crystal composition (iron content of the crystalline olivine grains). The $69 \mu \mathrm{m}$ band is not sensitive to the grain size (at grain sizes $\lesssim 7 \mu \mathrm{m}$ ), but is very sensitive to the grain temperature (for example see Suto et al. 2006; Koike et al. 2003; Sturm et al. 2010; de Vries et al. 2012).

The density structure of the dust around IRAS 17150-3224 was determined by Meixner et al. (2002) (see Sect. 4.2 and Table 2). We take their derived parameters for our radiative transport models and add forsterite to the model (see Table 2). The presence of forsterite in the outflow has no effect on the density or temperature structure and the results of Meixner et al. (2002), since the opacities of forsterite do not add any continuum opacity to the dust and only a small amount is needed to reproduce the $69 \mu \mathrm{m}$ band (see Table 2).

In order to extensively test all possible cases that could explain the features of IRAS 17150-3224, we explore five distributions for the forsterite, schematically depicted in the left column of Fig. 3:

- Model Whole: forsterite follows the same density distribution as the amorphous dust and is present in both the superwind and the low mass-loss rate AGB-wind previous to the superwind.

- Model Superwind: forsterite is only present in the superwind $\left(R<R_{\mathrm{SW}}\right)$.

- Model $A G B$-wind: forsterite is only present in the outflow that happened previous to the superwind $\left(R>R_{\mathrm{SW}}\right)$.

- Model Torus: forsterite is only present in the densest part of the superwind $\left(R<R_{\mathrm{SW}}, \theta>50^{\circ}\right)$

- Model Disk: forsterite is only present in a disk-like region in the superwind $\left(R<R_{\mathrm{SW}}, \theta>85^{\circ}\right)$.

Here $\theta$ is the angle from the pole and $R$ is the radial distance from the centre of the star. For all models, in the region where forsterite is present in the outflow, it follows the density distribution of the amorphous silicates (see Eq. (1)).

For these five models we test a grid of six grain sizes for forsterite: $\leq 0.1,1.0,2.0,4.0,6.0$ and $8.0 \mu \mathrm{m}$. We do not consider smaller grain sizes for forsterite since the absorption opacities do not change when the size is decreased from $0.1 \mu \mathrm{m}$ (see Fig. 2). For these different grain sizes we run a grid of models with different forsterite abundances from $0.5 \%$ up to $25 \%$ (for the Disk model only did we increase the abundance to $90 \%$ in order to get a signal at $69 \mu \mathrm{m}$ ). In these grids we search for the models with abundances that correctly predict the strength of the observed $69 \mu \mathrm{m}$ band. The abundances found for the different models are listed in Table 2. Among the models that reproduce the $69 \mu \mathrm{m}$ band we search for one that also explains the absence of the mid-infrared bands in the spectrum of IRAS 17150-3224.

\subsection{Models}

Meixner et al. (2002) fitted the SED up to $1.1 \mathrm{~mm}$ as well as the optical and near-IR resolved images of IRAS 17150-3224. It is beyond the scope of this work to improve on this already satisfactory model, and we adopt its parameters for the central star and the circumstellar environment (see Table 1). In Sect. 4.4, we do test the robustness of our results when uncertainties are introduced in the total dust mass and the stellar effective temperature determined by Meixner et al. (2002). Meixner et al. (2002) concluded that the best fit was obtained with an equatorially enhanced density distribution,

$$
\begin{aligned}
& \rho(R, \theta)=\rho_{\text {in }}\left(\frac{R}{R_{\text {in }}}\right)^{-B\left(1+C \sin ^{F}(\theta)\left(\mathrm{e}^{-\left(R / R_{\mathrm{SW}}\right)^{D}} / \mathrm{e}^{\left.\left.-\left(R_{\text {in }} / R_{\mathrm{SW}}\right)^{D}\right)\right)}\right.\right.} \\
& \times\left[1+A(1-\cos (\theta))^{F}\left(\mathrm{e}^{-\left(R / R_{\mathrm{SW}}\right)^{E}} / \mathrm{e}^{-\left(R_{\text {in }} / R_{\mathrm{SW}}\right)^{D}}\right)\right],
\end{aligned}
$$

with $R_{\text {in }}<R<R_{\mathrm{SW}} ; R$ is the distance from the centre of the central star and $\theta$ is the angle from the polar direction $(\theta=0)$; $R_{\text {in }}$ and $\rho_{\text {in }}$ are the inner radius and the density at the inner radius of the dust shell; and $R_{\mathrm{SW}}$ is the superwind radius outside of which the outflow is just spherically symmetric. Meixner et al. (2002) create a time-independent outflow (in all directions) by using an $r^{-2}$ density distribution $(B=2.0)$.

The parameters of the best-fit of Meixner et al. (2002) are shown in Table 1 . The total dust mass determined by Meixner et al. (2002) is $4.6 \times 10^{-2} M_{\odot}$. This mass is constrained by the shape and peak of the SED and the optical depth in the $9.7 \mu \mathrm{m}$ absorption band of amorphous silicates, in addition to reproducing the resolved images. The best fit of Meixner et al. (2002) required amorphous grains with an exponential cut-off at $200 \mu \mathrm{m}$ in size in order to reproduce the mm fluxes, as well as small amorphous grains (down to $0.001 \mu \mathrm{m}$ ) to fit the near and midinfrared part of the SED properly. For the central star we use a 
B. L. de Vries et al.: Micron-sized forsterite grains in the outflow of IRAS 17150
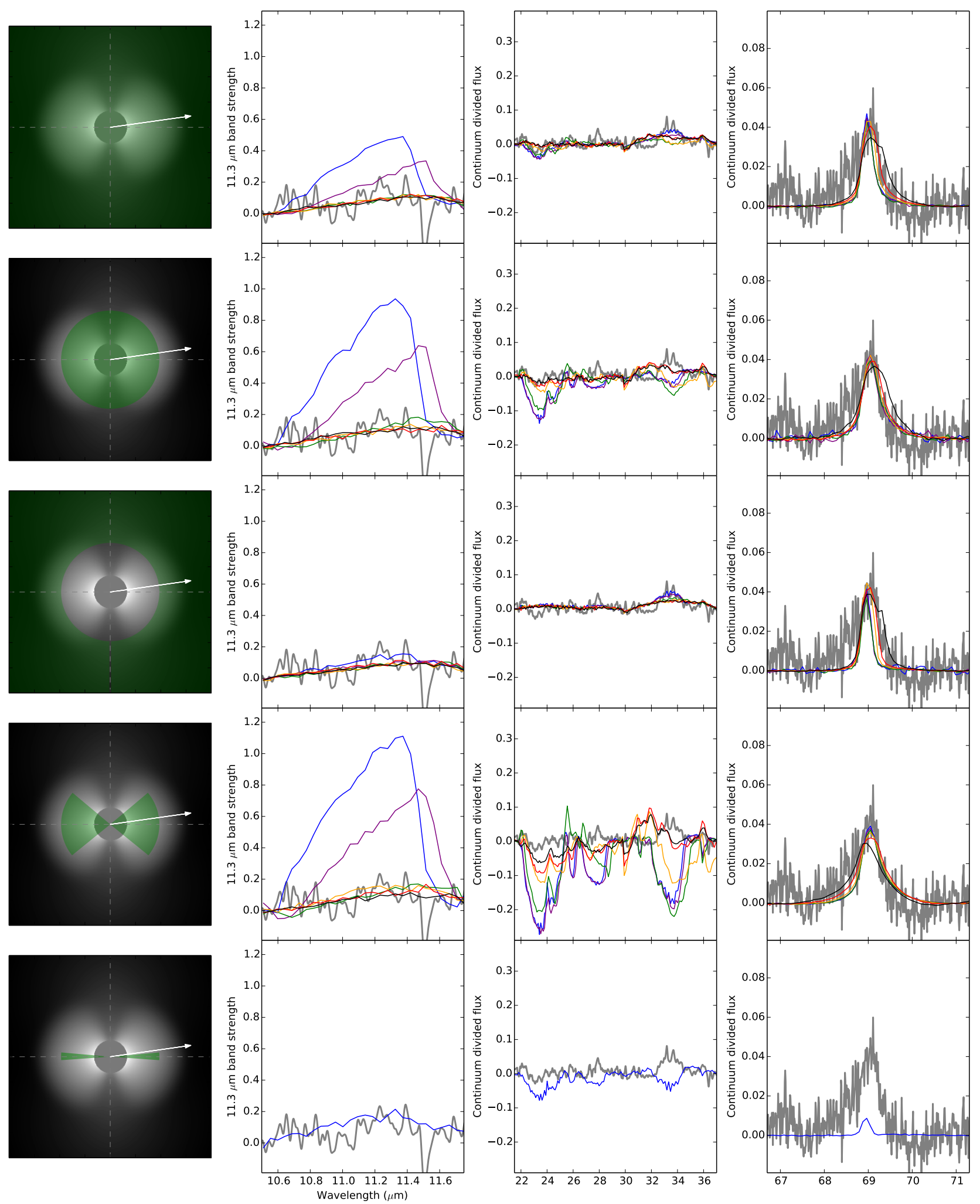

Fig. 3. Left column: density distributions (grey scale) and location of forsterite (green) for the different models described in Sect. 4.1. The white arrow shows the inclination of the system. Second and third columns: strength of the $11.3 \mu \mathrm{m}$ band and the other mid-infrared bands and fourth column: flux in the $69 \mu \mathrm{m}$ band of forsterite, all as described in Sect. 4.3. The different colours of the curves in the second, third and fourth column are for the observations (grey) and models with forsterite grains with different grain sizes: black $(0.1 \mu \mathrm{m})$, blue $(1.0 \mu \mathrm{m})$, red $(2.0 \mu \mathrm{m})$, orange $(4.0 \mu \mathrm{m})$, yellow $(6.0 \mu \mathrm{m})$, purple $(8.0 \mu \mathrm{m})$. The forsterite abundances are listed in Table 2.

temperature of $5200 \mathrm{~K}$. As shown by Sánchez Contreras et al. (2008) this temperature might be too low. Changes in the temperature of the central object will change the dust temperature in the inner region of the circumstellar environment. However, as shown in Sect. 4.4 and by de Vries et al. (2012), this will have no significant effects on the modelling of the dust in the case of very high optical-depth environments, because then the inner parts of the outflow are not directly observed. 
We have reproduced the best-fit model of Meixner et al. (2002) using the radiative transport code MCMax (Min et al. 2009). The MCMax code has been widely and successfully applied to model observables in a variety of environments (Mulders et al. 2011; Min et al. 2013; Lombaert et al. 2012, 2013). We refer to these papers for a full description of the features of MCMax. MCMax first computes the radiative equilibrium temperature stratification throughout the circumstellar environment. The temperature structure is calculated for the different grains present in the circumstellar environment separately, so no thermal coupling between different types of dust grains is assumed. For the size distribution of the amorphous grains, the opacity of grains with different sizes are weighted and combined. Using the resulting temperature structure, spectra can be obtained by ray-tracing. Dust scattering can be treated in an angle-dependent way, or, to speed up the computations (Min et al. 2009) by using isotropic scattering. In this work we use isotropic scattering.

We use the same size distribution and optical constants as Meixner et al. (2002) for the amorphous olivine $\left(\mathrm{MgFeSiO}_{4}\right)$, namely those of Dorschner et al. (1995), and we extend the model of Meixner et al. (2002) by including forsterite grains. MCMax uses temperature-dependent opacities, meaning the model correctly predicts the $69 \mu \mathrm{m}$ band shape depending on the temperature of the forsterite grains (Mulders et al. 2011). For crystalline olivine we use the optical constants of forsterite from Suto et al. (2006), which are available for grain temperatures of 50, 100, 150, 200 and $295 \mathrm{~K}$.

The opacities of the dust grains are calculated using the Distribution of Hollow Spheres (Min et al. 2003, $f_{\max }=1.0$ ). Other particle shape models used in the literature are spherical grains and the Continuous Distribution of Ellipsoids (CDE; Bohren \& Huffman 1983). We do not consider spherical grains because it has been shown that for crystalline dust species this model predicts spectral features with shapes and wavelength positions that do not compare with spectra of any astronomical object (Min et al. 2005). Min et al. (2005) also show that the DHS model reproduces the properties of forsterite spectral bands in spectra of astronomical objects very well. We also do not consider CDE grains for two reasons. The first is that it predicts very similar spectral features in shape and position as DHS (see Fig. 2). A difference between spectral features of CDE compared to $0.1 \mu \mathrm{m}$ DHS grains is that they are slightly weaker. Second, $\mathrm{CDE}$ is only valid for particles small compared to the wavelength of the radiation considered and does not allow us to model grains of different sizes, which is essential for this work.

\subsection{Extracting the forsterite spectral features}

A possible $11.3 \mu \mathrm{m}$ band would be situated on top of the red shoulder of the $9.7 \mu \mathrm{m}$ band of amorphous silicate, which is in absorption (see the inset of Fig. 1). The $11.3 \mu \mathrm{m}$ band has been extensively studied in massive AGB stars by de Vries et al. $(2010,2014)$. They study the ratio of the optical depth of forsterite relative to that of the amorphous continuum dust in the $9.7 \mu \mathrm{m}$ band. This ratio (here called the strength $S$ of the $11.3 \mu \mathrm{m}$ band) is interesting because in the optically thick limit it is proportional to the opacities (and abundance) of forsterite,

$$
S=\frac{\tau_{\mathrm{fo}}}{\tau_{\mathrm{all}}} \propto \frac{A_{\mathrm{fo}} \cdot \kappa_{\mathrm{fo}}}{\kappa_{\text {all }}},
$$

where $\tau_{\text {of }}$ and $\tau_{\text {all }}$ are the optical depth of forsterite only and the optical depth of all dust species together, respectively, and $A_{\mathrm{fo}}$ is the abundance of forsterite. Since the circumsteller environment of IRAS 17150-3224 is very dense and the optical depth

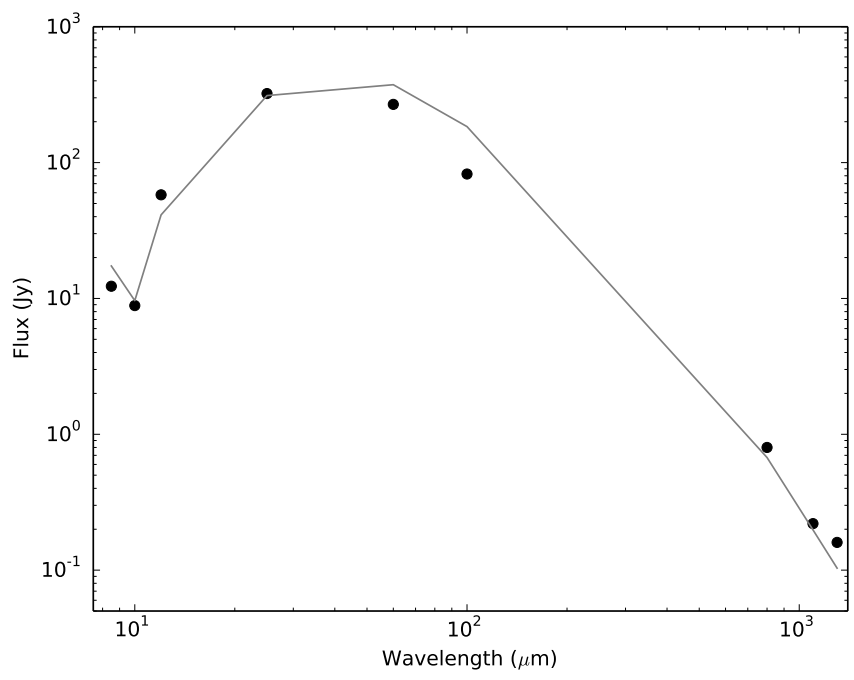

Fig. 4. Spectral energy distribution of IRAS 17150-3224 in black dots. The 8.5 and $10.0 \mu \mathrm{m}$ points are from Ueta et al. (2000), the 12, 25, 60, and $100 \mu \mathrm{m}$ points from IRAS, the 800.0 and $1100.0 \mu \mathrm{m}$ points from $\mathrm{Hu}$ et al. (1993) and the $1.3 \mathrm{~mm}$ from Guertler et al. (1996). In grey is our model based on the parameters of Meixner et al. (2002).

is much larger than one, Eq. (2) holds. This tells us that if there is forsterite in the line of sight to the star and the crystals do not approach a grain size of one micron, an absorption band of forsterite must be seen on the red shoulder of the $9.7 \mu \mathrm{m}$ band of amorphous olivine. If the forsterite grains are a micron or larger in size the strength $S$ will quickly go to zero.

We briefly describe how we measure the strength of the $11.3 \mu \mathrm{m}$ band (Eq. (2)) and refer to de Vries et al. (2010) for further details. In order to calculate the $\tau_{\text {all }}$ and $\tau_{\text {fo }}$ we first construct a continuum over the $9.7 \mu \mathrm{m}$ band of amorphous olivine (as shown in Fig. 1). The optical depth in the $9.7 \mu \mathrm{m}$ band is calculated using $\tau=-\ln \left(F / F_{\text {cont }}\right)$, where $F_{\text {cont }}$ is the flux level of the continuum constructed over the $9.7 \mu \mathrm{m}$ band of amorphous silicate. The optical depth in the $9.7 \mu \mathrm{m}$ band due to forsterite $\left(\tau_{\text {fo }}\right)$ and due to the other dust species $\left(\tau_{\text {all }}\right)$ is now determined by constructing a continuum in $\tau$-space, under the $11.3 \mu \mathrm{m}$ band (see again Fig. 1). For the $69 \mu \mathrm{m}$ band, we follow the approach of Blommaert et al. (2014) by fitting the continuum with a linear function (see Fig. 1). The mid-infrared bands are extracted in the region of 21 to $37 \mu \mathrm{m}$ by fitting a spline to the continuum points. The continuum points are taken at (all in $\mu \mathrm{m}$ ) 21.7-21.9; $26.5-26.7 ; 29.4-31.8 ; 36.7-37.8$.

\subsection{Base model and testing the results of Meixner et al. (2002)}

We find that our model SED, like the model SED of Meixner et al. (2002), matches the observed photometry well enough to study the spectral bands of forsterite (see Fig. 4). Similar to the model of Meixner et al. (2002), we slightly over-predict the flux at $100 \mu \mathrm{m}$, but due to the large amorphous grains added by Meixner et al. (2002) the fluxes around $1.0 \mathrm{~mm}$ are well reproduced. It is outside the scope of this work to try and improve the fit to the SED.

The spectral features we obtain by adding forsterite to the model with the best-fit parameters of Meixner et al. (2002) are shown in Fig. 3. We see that the model reproduces the observed $69 \mu \mathrm{m}$ band of IRAS $17150-3224$ well in strength, but its wavelength position is too blue and too narrow. This means 


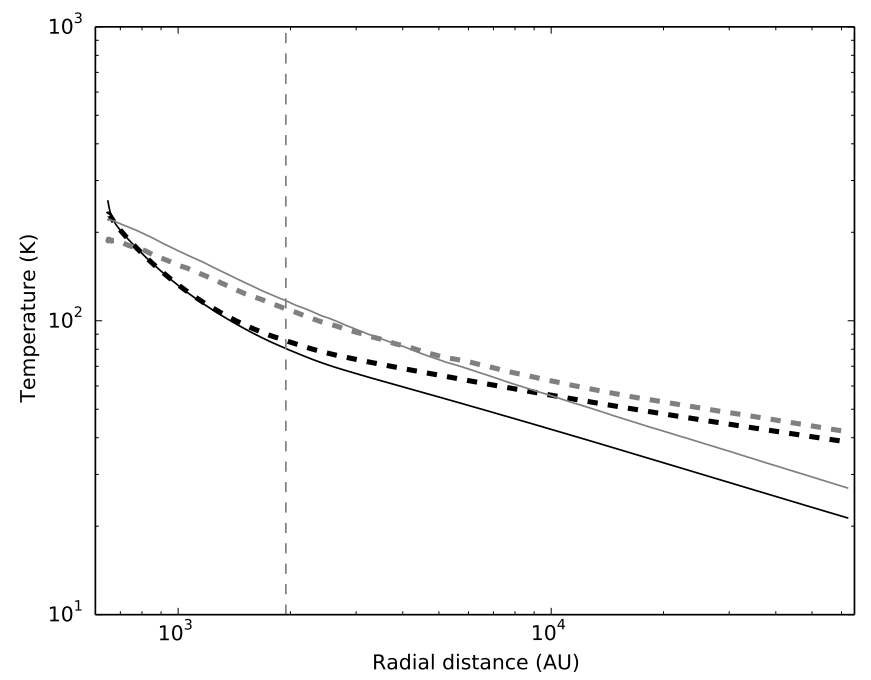

Fig. 5. Temperature gradient of the amorphous silicate (solid line) and the forsterite (dashed line) grains for the Whole model (see Sect. 4.1). Black lines give the temperature structure over the equator, while the grey gives it at the pole. The grey vertical dashed line indicates the transition between the low-density AGB wind and the superwind at the radius of $R_{\mathrm{SW}}=1944 \mathrm{AU}$.

that for this model the forsterite grains are too cold and/or too small. The mid-infrared bands of forsterite in the model spectrum are almost completely absent, similar to what is observed. The strength of the $11.3 \mu \mathrm{m}$ band on the other hand is too high in our model compared to the absence of any $11.3 \mu \mathrm{m}$ band in the observed spectrum. In Sect. 5 we will show what happens to the spectral features of forsterite if the forsterite grain size and geometrical distribution are varied.

Since different values for the total dust mass in the outflow and the central temperature of the star have been reported (Sánchez Contreras et al. 2008; Guertler et al. 1996) we want to test if a difference in these parameters has an effect on the spectral features of forsterite in the spectrum of IRAS 17150-3224. We investigate this by varying the central temperature and dust mass by $\pm 10 \%$. We found this has no significant effect on the spectral features. We therefore conclude that the fit of Meixner et al. (2002) and the parameters they obtained are of sufficient quality to reproduce the optical depth and temperature structure of the circumstellar environment in order for us to study the crystalline dust component in the outflow.

In Fig. 5 we show the equatorial and polar temperature gradient for both the amorphous dust and forsterite that we obtain with our model. We find that the temperature of the amorphous dust component, at the inner radius of the dust shell, reaches $\sim 220 \mathrm{~K}$, which is the same value as found by Meixner et al. (2002). The temperature gradients also show that at the equator and within the superwind $\left(R_{\mathrm{SW}}<1944 \mathrm{AU}\right)$ the optical depth is so high that the temperature of the dust is determined by the local radiation field of the dust. This means that both the amorphous dust and the forsterite have the same temperature. Outside the superwind the optical depth drops and the temperature structure of the amorphous dust and the forsterite starts to differ and the forsterite becomes slightly warmer than the amorphous dust. The forsterite is warmer than the amorphous dust because 1) the amorphous dust can cool more efficiently than forsterite since it has higher far-infrared opacities and 2) since both dust species are now heated by the radiation field of the inner dust shell. This shell radiates most of its energy in the mid-infrared, where forsterite has a higher overall absorption opacity. The optical depth at the pole is lower than at the equator and the forsterite is slightly cooler than the amorphous dust.

\section{Results}

The mid-infrared and $69 \mu \mathrm{m}$ bands of forsterite we find for the observations and models are shown in Fig. 3. In this section we will discuss the forsterite features of the five different models introduced in Sect. 4.1.

\section{Whole model}

In Fig. 3 it can be seen that the $11.3 \mu \mathrm{m}$ band in the Whole models for forsterite grain sizes of $\leq 0.1$ and $1.0 \mu \mathrm{m}$ are too strong. Only when the grain size is increased to $\geq 2 \mu \mathrm{m}$ does the $11.3 \mu \mathrm{m}$ band become weak enough and compare well with the observed absence of the $11.3 \mu \mathrm{m}$ band. The mid-infrared spectral bands do not strongly show in the models for any forsterite grain size. The $69 \mu \mathrm{m}$ band for models with forsterite grain sizes below $6 \mu \mathrm{m}$ have a wavelength position that is bluer and a shape that is more narrow than the observed band. The model with a forsterite grain size of $6.0 \mu \mathrm{m}$ reproduces the observed $69 \mu \mathrm{m}$ band well, while the $8 \mu \mathrm{m}$ forsterite grain size model has a too broad $69 \mu \mathrm{m}$ band.

\section{Superwind model}

Similar to the Whole model, the $11.3 \mu \mathrm{m}$ bands of the Superwind models are only weak enough for the models with forsterite grain sizes of $\geq 2 \mu \mathrm{m}$. The Superwind models with grain sizes of $\leq 2 \mu \mathrm{m}$ show absorption bands in the mid-infrared, especially at $\sim 23.5 \mu \mathrm{m}$. Only models with forsterite grain sizes of $\geq 4 \mu \mathrm{m}$ have weak enough mid-infrared bands to reproduce the observed spectrum. The $69 \mu \mathrm{m}$ bands of the Superwind models reproduce the observed band very well, except for the $69 \mu \mathrm{m}$ band in the model with $8 \mu \mathrm{m}$ sized forsterite grains. This model has a $69 \mu \mathrm{m}$ band that is too broad.

\section{AGB-wind model}

For this model the forsterite is so cold that no spectral features are seen at $11.3 \mu \mathrm{m}$ and in the mid-infrared for any forsterite grain size. Because the forsterite is so cold, the $69 \mu \mathrm{m}$ band is also very narrow and blue shifted and the models with forsterite grain sizes of $\leq 4 \mu \mathrm{m}$ do not reproduce the $69 \mu \mathrm{m}$ band well. At $8 \mu \mathrm{m}$ forsterite grain sizes the $69 \mu \mathrm{m}$ band is too broad, so only the $6 \mu \mathrm{m}$ forsterite grain size model reproduces the $69 \mu \mathrm{m}$ band well. The jagged feature on the red shoulder of the $69 \mu \mathrm{m}$ band for the $8 \mu \mathrm{m}$ forsterite grain size model occurs because the $69 \mu \mathrm{m}$ band develops a mild double-peaked shape at grain sizes of $\geq 8 \mu \mathrm{m}$ and low temperatures (Fig. 2).

\section{Torus model}

Similar to the Whole and Superwind model the Torus model with forsterite grain sizes of $\leq 2 \mu \mathrm{m}$ have a too strong $11.3 \mu \mathrm{m}$ band. In the mid-infrared the Torus models show absorption bands (especially at $23.5 \mu \mathrm{m}$ ) for all the forsterite grain sizes, except possibly for the $8 \mu \mathrm{m}$ model where the absorption features are weak enough to reproduce the observed absence of mid-infrared bands. All but the 6 and $8 \mu \mathrm{m}$ forsterite grain size models reproduce the $69 \mu \mathrm{m}$ band well for the Torus model. 


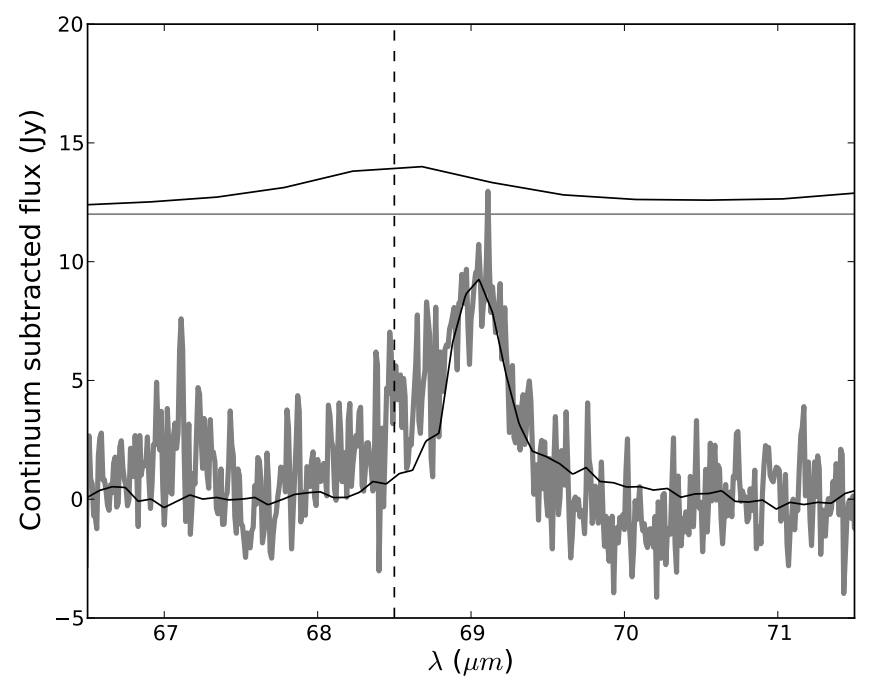

Fig. 6. Shown in grey is the observed, continuum subtracted $69 \mu \mathrm{m}$ band and over plotted in black a radiative transport model (Whole model, forsterite grain size of $4 \mu \mathrm{m}$ ). Shifted to the horizontal grey line are shown the scaled absorption opacities of ortho-enstatite (Chihara et al. 2002) in black. Ortho-enstatite has a resonance around $68.5 \mu \mathrm{m}$, indicated with a dashed black vertical line.

\section{Disk model}

We had to increase the forsterite abundance for this model to $90 \%$ in order to see a signal at $69 \mu \mathrm{m}$. It can be seen that even at this abundance the strength of the $69 \mu \mathrm{m}$ band cannot be reproduced.

\section{Blue shoulder on the $69 \mu \mathrm{m}$ band}

The centre and red shoulder of the $69 \mu \mathrm{m}$ band of IRAS 17150-3224 can be well reproduced by some of our models, while the small shoulder on the blue side is not (see also Fig. 6). The blue shoulder being located at roughly $68.5 \mu \mathrm{m}$ means it is too far to the blue side for it to be caused by a cold forsterite component (Suto et al. 2006; Koike et al. 2003). We suspect that this blue shoulder might be responsible for what caused Blommaert et al. (2014) to note that the $69 \mu \mathrm{m}$ band is too broad for its central wavelength position. An explanation can be the presence of ortho-enstatite $\left(\mathrm{MgSiO}_{3}\right.$; Chihara et al. 2002), which has a resonance at $68.5 \mu \mathrm{m}$. See Fig. 6 for a comparison of the blue shoulder and the opacities of ortho-enstatite. Orthoenstatite has another resonance at $72.5 \mu \mathrm{m}$, but this is in the middle of the two Herschel/PACS bands, making it difficult to study the presence of this spectral feature. Several resonances also occur in the mid-infrared, but they are not detected in the ISO-SWS spectrum. However, if the ortho-enstatite grains are of the same size as the forsterite grains, the mid-infrared bands would be suppressed as well. Since no other resonances are known to us around $68.5 \mu \mathrm{m}$, we find it likely that ortho-enstatite is present in the outflow of IRAS 17150-3224 with a grain size of a few micrometres.

\section{Summary}

We can exclude the Disk model since we cannot reproduce the $69 \mu \mathrm{m}$ band with an abundance as high as $90 \%$. We can also exclude forsterite grains of size $\leq 2 \mu \mathrm{m}$ for all models. For the Whole, Superwind and the Torus model this is based on the strength of the $11.3 \mu \mathrm{m}$ band. For the $A G B$-wind model this is based on the wavelength position of the $69 \mu \mathrm{m}$ band.

We can exclude forsterite grain sizes of $\geq 8 \mu \mathrm{m}$ because for all models the $69 \mu \mathrm{m}$ band becomes too broad. It is difficult to find a Torus model that does not have strong mid-infrared bands and we therefore find the Torus model unlikely. For the Whole, Superwind and $A G B$-wind case, satisfactory models can be found for forsterite grain sizes between $2 \mu \mathrm{m}$ and $6 \mu \mathrm{m}$.

\section{Discussion}

All of our models indicate micron-sized forsterite grains not smaller than $2 \mu \mathrm{m}$ and not larger than $6 \mu \mathrm{m}$. This shows that the forsterite is still significantly smaller than the largest amorphous grains $(\sim 200 \mu \mathrm{m})$. From dust condensation theory this difference can be understood, since the formation and growth of crystalline solids requires more specific conditions (Tielens et al. 1998; Gail \& Sedlmayr 1999; Sogawa \& Kozasa 1999). A high temperature (above $\sim 1000 \mathrm{~K}$ ) and pressure together with sufficient cooling times are needed to form a crystalline solid. Otherwise an amorphous solid is formed instead. In the outflows of evolved stars conditions for crystalline dust formation are only met in some cases, close to the central star in the inner part of the outflow. Because of this it can be understood that only small amounts of small crystals can be formed. This in contrast to amorphous solids, which can be formed and grown in larger regions of the outflow.

The fact that de Vries et al. (2014) can model consistently the $11.3 \mu \mathrm{m}$ and $69 \mu \mathrm{m}$ bands in massive AGB stars, means the forsterite crystals in the superwinds of these objects are sub-micron in size. We then find a large difference in forsterite grain size in superwinds of AGB stars and in the outflow of the PPN IRAS 17150-3224. At this moment there are no reports of large amorphous silicate grains in superwinds and the multitude of SED fits to superwind spectra in the far-infrared should have found large grains if they were present (Justtanont et al. 2006, 2013). For the massive AGB star $\mathrm{OH} 26.5+0.6$, Groenewegen (2012) finds an amorphous grain size between $0.15 \mu \mathrm{m}$ and $0.25 \mu \mathrm{m}$.

The high mm-fluxes of IRAS 17150-3224 together with our analysis of the forsterite spectral features show that there is an increase in grain size for both the amorphous and crystalline component in-between the superwind and the PPN phase. If there indeed exists a hyperwind, the conditions during it must lead to very efficient grain growth and crystallisation. We associate a very high density with the hyperwind and indeed dust condensation theory suggests that the density is critical in grain growth and the formation of crystalline silicates (Tielens et al. 1998; Gail \& Sedlmayr 1999; Sogawa \& Kozasa 1999). Besides the density, the temperature is also critical for the formation of crystalline solids. For olivine the temperature needs to be higher than its glass-temperature of $\sim 1000 \mathrm{~K}$, for it to condense into crystalline forsterite. Since we see no indication of a disk-like structure, the formation of the large forsterite crystals we see around IRAS 17150-3224 likely happened in the hot dust condensation region close to the star during the hyperwind, although we cannot exclude the $A G B$-wind model.

The firm lower-limit we find for the forsterite grain size is a challenge to explain. If the formation of crystalline material is efficient during the hyperwind, why is the forsterite grain size dominated by micron-sized crystals and why is there no population of sub-micron crystals? One possible mechanism we explored is based on the wind driving mechanism in oxygen-rich 


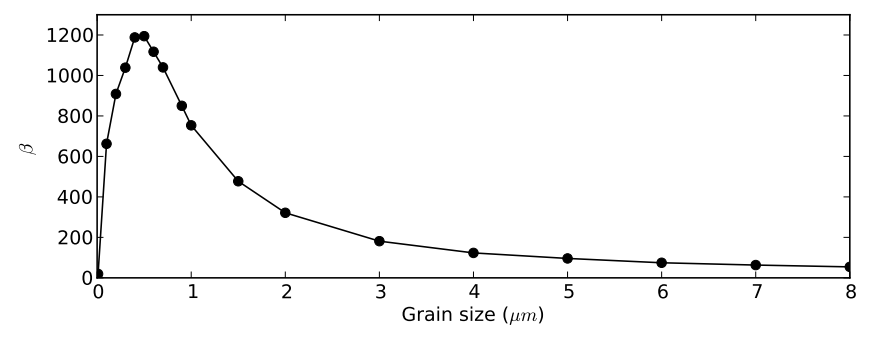

Fig. 7. Ratio of the radiation pressure over the gravitational force $(\beta)$ for forsterite grains of different grain sizes.

AGB outflows. The outflow of AGB winds is thought to be initiated by pulsations, which pushes gas to distances from the star where it can condense into dust grains (Wickramasinghe et al. 1966; Gehrz \& Woolf 1971; Sedlmayr 1994; Habing \& Olofsson 2003). Then radiation pressure on the most refractory dust grains drives the outflow to escape velocities. For carbon-rich AGB stars this works well (Winters et al. 2000; Gautschy-Loidl et al. 2004; Nowotny et al. 2010, 2011; Sacuto et al. 2011), but driving a wind by absorption of photons in an oxygen-rich environment is difficult (e.g. Jeong et al. 2003). This is because the refractory dust grains in an oxygen-rich environment (e.g. olivine and alumina) are transparent in the optical, meaning they absorb few photons, and thus momentum, from the radiation field of the central star (which has a temperature of $\sim 3000 \mathrm{~K}$ ). A suggestion to drive winds around oxygen-rich AGBs that seems to work well is momentum transfer to the grains by scattering (Höfner 2008; Bladh \& Höfner 2012). For this to be efficient, the grains need to grow to a size of $\sim 0.5 \mu \mathrm{m}$. Since forsterite is a refractory solid, it could be a driver of the outflow of massive AGB stars. We tested if the grain size dependence of the scattering efficiency of forsterite grains might be a way of grain-size-filtering, which might explain the lower limit for the grain size of forsterite around IRAS 17150-3224.

The radiative transport code MCMax that we use for the models (see Sect. 4.2) lets us calculate the $\beta$ of grains. This $\beta$ is the ratio of radiation pressure to gravitational force on the grain. The code takes into account the anisotropic scattering properties of the grains. A discussion on the driving of a superwind or a hyperwind is outside the scope of this paper, but we simply want to see how the $\beta$ of forsterite behaves as a function of grain size. Therefore we calculated the $\beta$ for forsterite grains at different grain sizes, shown in Fig. 7. We see, as shown before by Höfner (2008), that the momentum transfer by scattering becomes more efficient when the grains approach a size of $\sim 0.5 \mu \mathrm{m}$, but a maximum $\beta$ is also reached at $0.5 \mu \mathrm{m}$ and the scattering becomes less efficient at larger grain sizes. This is due to the decrease in the area to volume ratio of the grains. This shows that the lowerlimit we find for the forsterite grains around IRAS 17150-3224 is probably not directly linked to the wind driving by scattering on micron-sized grains. One could imagine that forsterite is the wind driving dust species and that a population of $\sim 0.5 \mu \mathrm{m}$ grains are blown out and that those subsequently grow to the observed size of $2-6 \mu \mathrm{m}$, but since the density quickly drops after the onset of the outflow, this would require very special circumstances.

Another possible scenario to explain the lower-limit of the forsterite grain size is that an initial population of forsterite grains is formed with a size distribution ranging from submicron grains up to $\sim 6 \mu \mathrm{m}$, but that the smaller grains are preferentially destroyed or amorphisised. If the smaller forsterite grains are preferentially destroyed, one has to explain why the small amorphous grains are not destroyed along with them. Instead of a destruction mechanism for both the amorphous and forsterite dust one could consider the selective amorphisation of forsterite grains instead.

One possible amorphisation mechanism is the destruction of the crystalline lattice structure by ion bombardments (for example $\mathrm{Ar}^{2+}$ or $\mathrm{Fe}^{2+}$; Borg et al. 1980; Day 1977; Bradley 1994; Demyk et al. 2001; Carrez et al. 2002; Jäger et al. 2003; Brucato et al. 2004; Kemper et al. 2004, 2005). Kemper et al. (2004) show that for the interstellar medium it takes several million years to destroy several percentage points by mass of sub-micron forsterite grains. Since the super- and hyperwind of IRAS 17150-3224 combined would not even take more than several thousand years, these amorphisation mechanisms are too slow. Possibly, more efficient amorphisation can be obtained at the inner-rim of the AGB (hyper) wind, where the fast stellar wind will impact the recently stopped hyperwind (Balick \& Frank 2002), but that could not easily explain the absence of small forsterite grains throughout the whole super and hyper outflow.

\section{Conclusions}

From studying the spectral features of forsterite in the spectrum of IRAS 17150-3224 we can draw the following conclusions:

- The forsterite crystals in the outflow are dominated by a grain size population between $2 \mu \mathrm{m}$ and $6 \mu \mathrm{m}$.

- For the forsterite component we cannot distinguish between the Whole, Superwind or AGB-wind models.

- We exclude the Torus and Disk models.

- The lower-limit for the forsterite grain size is likely unrelated to any wind driving mechanism based on scattering on micron-sized dust grains.

- The blue shoulder of the $69 \mu \mathrm{m}$ band cannot be explained by cold forsterite. We suggest the possibility of micron-sized ortho-enstatite as a carrier.

We also speculate that the following processes might be related to the hyperwind:

- The amorphous grains grow to several times $100 \mu \mathrm{m}$ in size during the hyperwind.

- Forsterite grains can grow up to sizes as large as $\sim 6 \mu \mathrm{m}$ during the hyperwind.

- The absence of sub-micron forsterite grains could be due to an amorphisation mechanism currently unknown.

\section{References}

Balick, B., \& Frank, A. 2002, ARA\&A, 40, 439

Bedijn, P. J. 1987, A\&A, 186, 136

Bladh, S., \& Höfner, S. 2012, A\&A, 546, A76

Blommaert, J. A. D. L., de Vries, B. L., Waters, L. B. F. M., et al. 2014, A\&A, $565, \mathrm{~A} 109$

Bohren, C. F., \& Huffman, D. R. 1983, Absorption and scattering of light by small particles (New York: Wiley)

Borg, J., Chaumont, J., Langevin, Y., Maurette, M., \& Jouret, C. 1980, in The Ancient Sun: Fossil Record in the Earth, Moon and Meteorites, eds. R. O. Pepin, J. A. Eddy, \& R. B. Merrill, 431

Bowey, J. E., Barlow, M. J., Molster, F. J., et al. 2002, MNRAS, 331, L1

Bradley, J. P. 1994, Science, 265, 925

Brucato, J. R., Strazzulla, G., Baratta, G., \& Colangeli, L. 2004, A\&A, 413, 395

Carrez, P., Demyk, K., Cordier, P., et al. 2002, Meteor. Planet. Sci., 37, 1599

Chesneau, O., Verhoelst, T., Lopez, B., et al. 2005, A\&A, 435, 563

Chihara, H., Koike, C., Tsuchiyama, A., Tachibana, S., \& Sakamoto, D. 2002, A\&A, 391, 267

Cox, N. L. J., Kerschbaum, F., van Marle, A.-J., et al. 2012, A\&A, 537, A35 
Day, K. L. 1977, MNRAS, 178, 49P

de Vries, B. L., Min, M., Waters, L. B. F. M., Blommaert, J. A. D. L., \& Kemper, F. 2010, A\&A, 516, A86

de Vries, B. L., Acke, B., Blommaert, J. A. D. L., et al. 2012, Nature, 490, 74

de Vries, B. L., Blommaert, J. A. D. L., Waters, L. B. F. M., et al. 2014, A\&A, 561, A75

Decin, L., Hony, S., de Koter, A., et al. 2007, A\&A, 475, 233

Demyk, K., Carrez, P., Leroux, H., et al. 2001, A\&A, 368, L38

Dijkstra, C., Waters, L. B. F. M., Kemper, F., et al. 2003, A\&A, 399, 1037

Dorschner, J., Begemann, B., Henning, T., Jaeger, C., \& Mutschke, H. 1995, A\&A, 300, 503

Gail, H.-P., \& Sedlmayr, E. 1999, A\&A, 347, 594

Gautschy-Loidl, R., Höfner, S., Jørgensen, U. G., \& Hron, J. 2004, A\&A, 422, 289

Gehrz, R. D., \& Woolf, N. J. 1971, ApJ, 165, 285

Groenewegen, M. A. T. 1994, A\&A, 290, 544

Groenewegen, M. A. T. 2012, A\&A, 543, A36

Groenewegen, M. A. T., Waelkens, C., Barlow, M. J., et al. 2011, A\&A, 526, A 162

Guertler, J., Koempe, C., \& Henning, T. 1996, A\&A, 305, 878

Habing, H. J., \& Olofsson, H. 2003, Asymptotic giant branch stars (New York, Berlin: Springer)

Heske, A., Forveille, T., Omont, A., van der Veen, W. E. C. J., \& Habing, H. J. 1990, A\&A, 239, 173

Höfner, S. 2008, A\&A, 491, L1

Hu, J. Y., Slijkhuis, S., Nguyen-Q-Rieu, \& de Jong, T. 1993, A\&A, 273, 185

Iben, Jr., I., \& Renzini, A. 1981, Physical processes in red giants, Proc. of the

Second Workshop, Advanced School of Astronomy, Erice, Italy, September 3-13 1980, Astrophys. Space Sci. Lib., 88

Jäger, C., Fabian, D., Schrempel, F., et al. 2003, A\&A, 401, 57

Jeong, K. S., Winters, J. M., Le Bertre, T., \& Sedlmayr, E. 2003, A\&A, 407, 191

Justtanont, K., \& Tielens, A. G. G. M. 1992, ApJ, 389, 400

Justtanont, K., Skinner, C. J., Tielens, A. G. G. M., Meixner, M., \& Baas, F. 1996, ApJ, 456, 337

Justtanont, K., Olofsson, G., Dijkstra, C., \& Meyer, A. W. 2006, A\&A, 450, 1051

Justtanont, K., Teyssier, D., Barlow, M. J., et al. 2013, A\&A, 556, A101

Kemper, F., Vriend, W. J., \& Tielens, A. G. G. M. 2004, ApJ, 609, 826

Kemper, F., Vriend, W. J., \& Tielens, A. G. G. M. 2005, ApJ, 633, 534

Knapp, G. R., \& Morris, M. 1985, ApJ, 292, 640

Koike, C., Chihara, H., Tsuchiyama, A., et al. 2003, A\&A, 399, 1101

Kwok, S., Hrivnak, B. J., Zhang, C. Y., \& Langill, P. L. 1996, ApJ, 472, 287
Lombaert, R., de Vries, B. L., de Koter, A., et al. 2012, A\&A, 544, L18

Lombaert, R., Decin, L., de Koter, A., et al. 2013, A\&A, 554, A142

Maaskant, K. M., de Vries, B. L., Min, M., et al. 2015, A\&A, 574, A140

Maercker, M., Mohamed, S., Vlemmings, W. H. T., et al. 2012, Nature, 490, 232

Meixner, M., Ueta, T., Dayal, A., et al. 1999, ApJS, 122, 221

Meixner, M., Ueta, T., Bobrowsky, M., \& Speck, A. 2002, ApJ, 571, 936

Min, M., Hovenier, J. W., \& de Koter, A. 2003, in Conf. Electromagnetic and Light Scattering - Theory and Applications VII, 230

Min, M., Hovenier, J. W., \& de Koter, A. 2005, A\&A, 432, 909

Min, M., Dullemond, C. P., Dominik, C., de Koter, A., \& Hovenier, J. W. 2009, A\&A, 497, 155

Min, M., Jeffers, S. V., Canovas, H., et al. 2013, A\&A, 554, A15

Molster, F. J., Waters, L. B. F. M., Tielens, A. G. G. M., \& Barlow, M. J. 2002, A\&A, 382, 184

Mulders, G. D., Waters, L. B. F. M., Dominik, C., et al. 2011, A\&A, 531, A93

Murakawa, K., Izumiura, H., Oudmaijer, R. D., \& Maud, L. T. 2013, MNRAS, 430, 3112

Nowotny, W., Höfner, S., \& Aringer, B. 2010, A\&A, 514, A35

Nowotny, W., Aringer, B., Höfner, S., \& Lederer, M. T. 2011, A\&A, 529, A129

Sacuto, S., Aringer, B., Hron, J., et al. 2011, A\&A, 525, A42

Sánchez Contreras, C., Sahai, R., Gil de Paz, A., \& Goodrich, R. 2008, ApJS, 179,166

Schutte, W. A., \& Tielens, A. G. G. M. 1989, ApJ, 343, 369

Sedlmayr, E. 1994, in IAU Colloq. 146: Molecules in the Stellar Environment, ed. U. G. Jorgensen (Berlin: Springer Verlag), Lect. Notes Phys., 428, 163

Sloan, G. C., Kraemer, K. E., Price, S. D., \& Shipman, R. F. 2003, ApJS, 147, 379

Sogawa, H., \& Kozasa, T. 1999, ApJ, 516, L33

Sturm, B., Bouwman, J., Henning, T., et al. 2010, A\&A, 518, L129

Sturm, B., Bouwman, J., Henning, T., et al. 2013, A\&A, 553, A5

Suto, H., Sogawa, H., Tachibana, S., et al. 2006, MNRAS, 370, 1599

Tielens, A. G. G. M., Waters, L. B. F. M., Molster, F. J., \& Justtanont, K. 1998, Ap\&SS, 255, 415

Ueta, T., Meixner, M., \& Bobrowsky, M. 2000, ApJ, 528, 861

van der Veen, W. E. C. J., Habing, H. J., \& Geballe, T. R. 1989, A\&A, 226, 108 Vassiliadis, E., \& Wood, P. R. 1993, ApJ, 413, 641

Waters, L. B. F. M., Molster, F. J., de Jong, T., et al. 1996, A\&A, 315, L361

Wickramasinghe, N. C., Donn, B. D., \& Stecher, T. P. 1966, ApJ, 146, 590

Winters, J. M., Le Bertre, T., Jeong, K. S., Helling, C., \& Sedlmayr, E. 2000, A\&A, 361, 641

Wood, P. R., Whiteoak, J. B., Hughes, S. M. G., et al. 1992, ApJ, 397, 552

Zeidler, S. 2012, EAS Pub. Ser., 58, 409 